\title{
Funcionamiento familiar en pacientes drogodependientes
}

\section{Family functioning in drug dependent patients}

\section{Funcionamiento familiar}

Freya Erika López Vivas ${ }^{(1)}$

\section{Hernán Cedeño ${ }^{(2)}$}

${ }^{1}$ Instituto de Posgrado de la Universidad Técnica de Manabi. Ecuador flopez6681@utm.edu.ec. Código Orcid https://orcid.org/0000-0001-5681-6613

${ }^{2}$ Instituto de Posgrado de la Universidad Técnica de Manabi. Ecuador. hernan.cedeno@utm.edu.ec Código Orcid orcid.org/0000-0002-3178-953X

Contacto: flopez6681@utm.edu.ec

\section{Resumen}

El objetivo de la presente investigación fue establecer el nivel actual de funcionamiento familiar de los pacientes drogodependientes. La investigación fue cuantitativa de tipo descriptiva, para la recolección de información se aplicó un cuestionario de APGAR familiar, de sus siglas en inglés Adaptability (Adaptación), Participation (Participación), Growth (crecimiento), Affected (Afecto), Resources (Recursos). a los centros especializados en el consumo de alcohol y drogas CENTRADRO y Camino a la paz. Los resultados señalaron que dentro de una de los centros el nivel de funcionalidad es alto. Se logró concluir que establecer el nivel de funcionalidad familiar permitirá trabajar aspectos centrales que ayuden a fortalecer el desarrollo integral de los pacientes drogodependientes.

Palabras clave: Drogas, consumo, familia

\section{Sumary}

The objective of the present investigation was to establish the current level of family functioning of drugdependent patients. The research was quantitative and descriptive. To collect information, a family APGAR 
(Adaptabilit, Participation, Growth, Affected, Resources) questionnaire was applied to two centers specialized in the consumption of alcohol and drugs. The results indicated that within one of the centers the level of functionality is high. It was concluded that establishing the level of family function will allow to work on central aspects that help to strengthen the comprehensive development of drug-dependent patients

Keyword: drugs, consumption, family

\section{Introducción}

La familia constituye un sistema, por lo tanto, lo que le afecte a uno de los miembros afecta a todo este sistema familiar. Visto de este modo, el consumo de sustancias es signo de que el sistema está afectado y esto trae consigo afectaciones en el desarrollo del individuo y sus áreas de funcionamiento.

La importancia de la aplicación de un modelo psicoterapéutico familiar sistémico en pacientes drogodependientes radica en que no solo involucra un cambio individual del paciente sino a todo el contexto familiar. Así, se estima que consiga disminuir aspectos como la tensión familiar, formando cohesión en el grupo, fortaleciendo la relación entre los miembros que ayudara a aumentar el compromiso de los pacientes y sus familias con el programa de intervención.

Mediante el presente estudio se evidencia información relevante sobre el funcionamiento familiar, el consumo de sustancias y la relación en los mismos, para así, dar un aporte a mejorar la calidad de vida de los mismos y sus familias. Todo esto con el fin de que los resultados sean de base para futuras investigaciones y sirvan de ayuda a profesionales inmersos en este campo.

La terapia familiar estructural centrara su análisis en el comportamiento familiar, en la dinámica de cada integrante con el grupo. El profesional o terapeuta deberá trabajar en la interacción, limites y funcionamiento de la familia llegando a consensos con cada uno de ellos. El objetivo del profesional será reorganizar a la familia destacando sus fortalezas y así logrando el cambio integral (Jaramillo, 2016).

Es por esto se hace importante mencionar la importancia de la terapia familiar ya que no solo involucra un cambio individual del paciente sino en todo el contexto familiar lo cual hará como efecto rebote un cambio a cada uno de los individuos dentro del sistema, siendo así el Dr. Carlos Nolasco Pereyra esquematiza diferencias básicas entre la terapia individual y terapia familiar en el cual describe: "La relación tiene como propósito definido Cambios en la conducta (y/o estructura) de uno de cambios en el sistema familiar de interacción, no el sus participantes. De cada uno de sus individuos. “ (Nolasco Pereyra, pág. 295) 
En una investigación realizada en España con el tema La Terapia Familiar en el tratamiento de las adicciones los autores describen la importancia de la terapia familiar en el trabajo terapéutico con el paciente mediante revisión literaria y dentro de su conclusión (Marcos Sierra \& Garrido Fernandez, 2009) exponen:

"existe una amplia evidencia que demuestra la eficacia de las intervenciones familiares en el tratamiento de las adicciones...Si a esto añadimos que las actuales tendencias de tratamiento tienen cada vez más componentes de tipo ambulatorio y por consiguiente una mayor presencia de la familia," pag.359

Por otro lado en Guatemala en un estudio realizado con el tema Terapia Familiar y Adicciones Un enfoque práctico con resultados prácticos el autor concluye "Los resultados señalan que es más probable que si la familia enfrenta el problema en forma conjunta, las recaídas son menores que el abstenerse del consumo de sustancias" (Ramirez Villaseñor, 2001, pág. 9) es decir se resalta la importancia que recae en la familia para el proceso del paciente

En nuestro país se realizó en Machala un proyecto con tema Plan terapéutico para adicciones basadas en terapia cognitivo-conductual se recalca el valor de la familia en temas adictivos "Las relaciones familiares son fundamentales como apoyo primordial para quienes experimentan casos adictivos” (Moreno Alvarado, 2016, pág. 33)

Al ser un sistema la familia se verá afectada si una de las partes falla algo, por ello el conflicto de uno de los integrantes se verá reflejado en la familia, por ello nos vemos en la necesidad de incluirlos, aunque ellos no tengan problemas con el alcohol y/o drogas son los que reciben el síntoma de lo que sucede, la intervención familiar lograra desintoxicar a la familia y generar más confianza en ellos (Rodriguez, 2018).

Como consecuencia de un estudio en el cual usaron la terapia familiar estratégica breve en la rehabilitación de adolescente con consumo de alcohol que se hallaron internos en el centro se pudo corroborar que las recaídas disminuyeron, a razón de que la familia junto con el paciente se siente cómodos, la confianza aumenta y solucionan situaciones personales y familiares (Luna, 2015).

A pesar de que puedan existir barreras en recibir intervención familiar entre ellas la inexistencia del apoyo familiar, familia con consumo de alcohol y/o drogas, patologías duales, una vez aceptada la intervención profesional se logra que el paciente y su familia conozcan los factores de protección que le servirán en el afecto y la comunicación que serán útiles para reducir la adicción (Zapata, 2009). 
Cabe destacar que no es tarea fácil el incluir a la familia dentro del proceso, pero siempre será importante, por ello se deberá trabajar de manera conjunta para lograr metas terapéuticas, cuando se trata de menores de edad los padres se deberán hacer cargo de la vida personal del paciente para que supere su adicción, en los adultos la meta es que lo aprenda a hacer solo, aunque con la ayuda familiar (Castillo et al., 2018).

La terapia familiar sistémica tiene eficacia en problemas de alcohol y/o drogas y se fundamentan en bases como se menciona: "Los desarrollos iniciales establecieron las bases sobre las cuales se implementan hoy en día los trabajos terapéuticos con estas familias, fundamentalmente mediante dos tipos de intervenciones: estructurales o estratégicas/paradójicas." (Cócola, 2018).

Basado en datos estadísticos de un estudio realizado en Trujillo, se comprobó que la funcionalidad de los pacientes que recibieron en su proceso terapia familiar era del $85 \%$ y los que no recibieron dicha terapia en el proceso del $25 \%$, en lo referente a las recaídas los que reciben terapia $20 \%$, y los que no $77.5 \%$. estos resultados permiten conocer que estas terapias se convierten en un factor de protección para el paciente (Sanchez \& Serrano, 2019).

La familia se presenta en la vida de cada persona como un lugar de encuentro, conocimiento propio, formación en valores y crecimiento personal. Sin embargo, existen situaciones que afectan el funcionamiento de la familia (Bustos \& Nossa, s. f.).

Al ser la familia parte del proceso de aprendizaje del individuo, será ejemplo desde su nacimiento, tanto de conductas adaptativas que ayudaran a su nutrición personal como conductas conflictivas, mismas conductas que pueden desaprenderse con el pasar del tiempo y aprenderse nuevas en su lugar (Costa, 2014)

El conjunto de patrones de relación que se dan entre los integrantes de la familia durante las etapas de su desarrollo integral, estableciendo roles que se ven influenciados por ambientes sociales con los que han venido interactuando se lo denomina funcionamiento familiar.(García et al., 2006)

Los instrumentos que ofrecen una apreciación de la relación familiar, permiten desarrollar una visión integrada de las interacciones familiares actuales, puntos débiles y fuertes de la familia, convirtiéndose así en elementos importante de diagnóstico para el médico familiar (Alegre \& Suarez, 2006).

Para llegar a determinar el nivel de funcionamiento familiar se han utilizados diversos instrumentos dentro de los cuales se encuentra el cuestionario de APGAR familiar creado por el Dr. Gabriel Smilkstein, compuesto por 5 ítems que miden adaptabilidad, cooperación, desarrollo, afectividad y capacidad resolutiva. 
La aplicación del Apgar familiar promueve el interés y expresión de sentimientos entre el paciente y el profesional a cargo, permite la comprensión del entorno familiar, guiando a buscar estrategias que puedan mejorar el funcionamiento familiar.(Suarez, 2014)

Así también permite identificar las funciones básicas familiares y que rol desempeña cada uno de los integrantes de la familia en el proceso de recuperación, lo que representan una ayuda indispensable para determinar factores de debilidad en el entorno familiar en la atención de salud en general.(Suarez, 2014)

El componente adaptación, del APGAR familiar, está basado en la resolución de los problemas cuando el equilibrio de la familia se ve amenazado por un conflicto durante un período de crisis(Ortega et al., 2003).

La presente investigación tuvo como objetivos establecer el nivel actual de funcionamiento familiar de los pacientes drogodependientes, Determinar la diferencia entre los pacientes drogodependientes del CETAD "CENTRADRO" Y "Camino a la paz" en cuanto el funcionamiento familiar y la edad de los pacientes y Determinar la diferencia entre los pacientes drogodependientes del CETAD “CENTRADRO” Y "Camino a la paz" en cuanto el funcionamiento familiar y el nivel académico.

\section{Materiales y métodos}

La presente investigación fue cuantitativa de tipo descriptivo mediante la aplicación del cuestionario de APGAR familiar, mediante el procesamiento de la información se dio mediante el programa SPSS, la población fue de 34 pacientes internos en Centros especializados en tratamiento de alcohol y drogas, donde se trabajó con 21 pacientes internos del CETAD “CENTRADRO” y 11 pacientes del CETAD “Camino a la paz", se incluyeron a los pacientes mayores de 18 años, hombres, con consumo problemático de drogas y familia de tipo nuclear, se excluyeron de la misma a los pacientes que sus familias no participan en el proceso de rehabilitación, que no firmaron el consentimiento informado y con patologías duales, los participantes firmaron los respectivos consentimientos informados. Declararon no hay conflicto de interés y confidencialidad de la información.

\section{Resultados}

Presentación y análisis que se obtuvo de los datos del cuestionario de APGAR familiar aplicado a los pacientes internos en los Centros Especializado en Tratamiento de alcohol y drogas "Camino a la paz" y “CENTRADRO”. 
Tabla 1. Porcentaje de media, mediana, moda y validez de los datos del funcionamiento familiar de los internos del Centro Especializado en Tratamiento de alcohol y drogas "Camino a la paz" 2021

\section{Estadísticos}

Funcionalidad

\begin{tabular}{lcc} 
& Válido & 12 \\
\cline { 2 - 3 } & Perdidos & 0 \\
\hline Media & 1,5000 \\
\hline Mediana & 1,0000 \\
\hline Moda & 1,00
\end{tabular}

Siendo 1 el porcentaje equivalente a un nivel funcional, lo que representa la moda es equivalente a que la mayor parte de participantes tienen un nivel funcional, siendo así, este nivel funcional determinado ayuda al terapeuta a fomentar otras áreas importantes en la relación familiar basado en esta funcionalidad presente en los participantes.

Tabla 2. Porcentaje de funcionalidad familiar de los internos del Centro Especializado en Tratamiento de alcohol y drogas "Camino a la paz" 2021

\section{Funcionalidad}

\begin{tabular}{|c|c|c|c|c|c|}
\hline & & Frecuencia & Porcentaje & $\begin{array}{c}\text { Porcentaje } \\
\text { válido }\end{array}$ & $\begin{array}{l}\text { Porcentaje } \\
\text { acumulado }\end{array}$ \\
\hline \multirow[t]{4}{*}{ Válido } & Funcional & 7 & 58,3 & 58,3 & 58,3 \\
\hline & Disfuncional leve & 4 & 33,3 & 33,3 & 91,7 \\
\hline & $\begin{array}{l}\text { Disfuncional } \\
\text { grave }\end{array}$ & 1 & 8,3 & 8,3 & 100,0 \\
\hline & Total & 12 & 100,0 & 100,0 & \\
\hline
\end{tabular}


Mediante la recolección de datos a los 12 pacientes se determina que los participantes en su mayoría presentan un nivel funcional con un 58,3\% con una frecuencia de 7, con una frecuencia de 4 equivalente al 33,3\% disfuncionalidad leve, mientras que el $8,3 \%$ con una frecuencia de 1 se presenta un nivel disfuncional grave es decir solo uno de los participantes percibe la relación familiar por debajo de lo normal, el estar pasando por un proceso terapéutico ayuda a los mismos a identificar ciertos aspectos familiares que causan malestar como es en el caso de los participantes que presentan un nivel disfuncional pero así también son capaces de reconocer aspectos integradores de la familia como en el caso de quienes presentaron un nivel funcional.

Tabla 3. Cruzada de datos de edad y nivel de funcionalidad de los internos del Centro Especializado en Tratamiento de alcohol y drogas "Camino a la paz" 2021

\section{Tabla cruzada Edad*Funcionalidad}

\begin{tabular}{|c|c|c|c|c|c|}
\hline & \multicolumn{4}{|c|}{ Funcionalidad } & \multirow[b]{2}{*}{ Total } \\
\hline & & Funcional & Disfuncional leve & Disfuncional grave & \\
\hline \multirow[t]{3}{*}{$\overline{\text { Edad }}$} & $18-27$ & 4 & 4 & 1 & 9 \\
\hline & $28-37$ & 2 & 0 & 0 & 2 \\
\hline & $38-47$ & 1 & 0 & 0 & 1 \\
\hline Total & & 7 & 4 & 1 & 12 \\
\hline
\end{tabular}

Mediante la recolección de datos a los 12 pacientes se determina que el mayor número de participantes se encuentran entre las edades de 18 a 21 años representados entre un nivel de funcionalidad que va desde disfuncional leve a funcional mientras que estando dentro de este mismo nivel de edad se presenta 1 participante con disfuncionalidad grave. El consumo de sustancias se ve representado en un mayor nivel en pacientes con edades equivalentes la adultez joven, dicha edad que comprende la integración y participación dentro de un grupo tanto con familia como compañeros como también pueden verse aislados frente a los mismos, es por esto que dentro de esta categoría de edad todas las categorías de los niveles de funcionalidad de ven representadas. 
Tabla 4. Cruzada de datos de nivel académico y nivel de funcionalidad de los internos del Centro Especializado en Tratamiento de alcohol y drogas "Camino a la paz" 2021

\section{Tabla cruzada Nivel Académico*Funcionalidad}

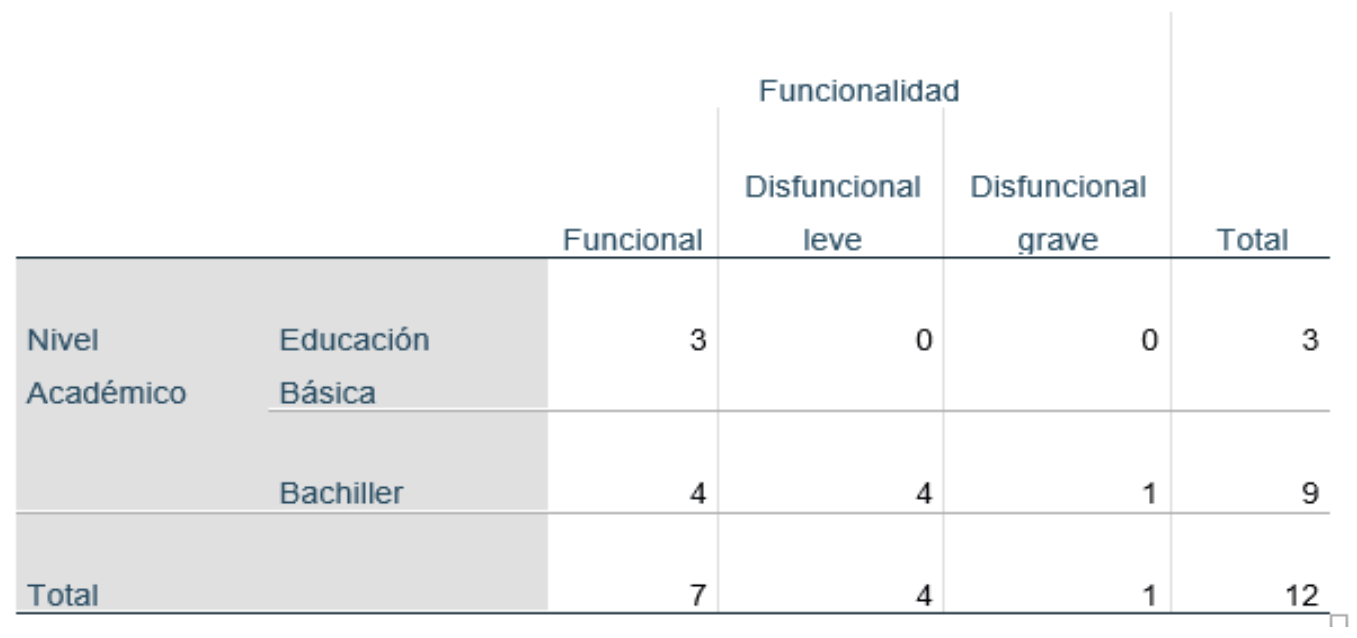

Mediante la recolección de datos a los 12 pacientes se determina que el mayor número de participantes culminaron sus estudios secundarios como bachilleres de la república del Ecuador, representados con 4 un nivel funcional y 4 con nivel disfuncionalidad leve, dentro de este mismo rango 1 representa disfuncionalidad grave, mientras que dentro del rango Educación básica se encuentran 3 participantes con un nivel funcional, el consumo afecta varias áreas del desarrollo personal del paciente entre ellas está la educación, siendo así que dentro de los datos recolectados se ha perdido el rango de educación superior debido a que ningún participante de los 12 entran en este nivel.

Tabla 5. Porcentaje de media, mediana, moda y validez de los datos del funcionamiento familiar de los internos del Centro Especializado en Tratamiento de alcohol y drogas “CENTRADRO” 2021

\section{Estadísticos}

Funcionalidad

\begin{tabular}{ll} 
Válido & 21 \\
\cline { 2 - 2 } Perdidos & 0 \\
\hline
\end{tabular}




\begin{tabular}{lc}
\hline Media & 1,6667 \\
\hline Mediana & 2,0000 \\
\hline Moda & 2,00 \\
\hline
\end{tabular}

Siendo 2 el porcentaje equivalente a un nivel disfuncional leve, lo que representa la moda es equivalente a que la mayor parte de participantitas tienen un nivel disfuncional leve, el pasar bajo un proceso de rehabilitación los pacientes adquieren habilidades que permiten reconocer factores de conflicto dentro de sus áreas de funcionamiento, en este caso representaría su área familiar, determinando así mediante la aplicación del cuestionario con un nivel disfuncional leve.

Tabla 6. Porcentaje de funcionalidad familiar de los internos del Centro Especializado en Tratamiento de alcohol y drogas “CENTRADRO” 2021

\section{Funcionalidad}

\begin{tabular}{|c|c|c|c|c|c|}
\hline & & Frecuencia & Porcentaje & $\begin{array}{l}\text { Porcentaje } \\
\text { válido }\end{array}$ & $\begin{array}{l}\text { Porcentaje } \\
\text { acumulado }\end{array}$ \\
\hline \multirow[t]{3}{*}{ Válido } & Funcional & 7 & 33,3 & 33,3 & 33,3 \\
\hline & Disfuncional leve & 14 & 66,7 & 66,7 & 100,0 \\
\hline & Total & 21 & 100,0 & 100,0 & \\
\hline
\end{tabular}

Mediante la recolección de datos a los 21 pacientes se determina que los participantes en su mayoría presentan un nivel disfuncional leve con un 66,7\% que representa una frecuencia de 14 mientras que el 33,3\% que representa una frecuencia de 7 apunta a un nivel funcional, quedándose fuera el nivel disfuncional grave, a esto el pasar por un proceso terapéutico vivencial que brindan los centros especializados en tratamiento de alcohol y drogas permiten al paciente percibir aspectos positivos de su entorno familiar que bajo los efectos del consumo no eran percibidos.

Tabla 7. Cruzada de datos de edad y nivel de funcionalidad de los internos del Centro Especializado en Tratamiento de alcohol y drogas "CENTRADRO” 2021 


\section{Tabla cruzada Edad*Funcionalidad}

\begin{tabular}{|c|c|c|c|c|}
\hline & & \multicolumn{2}{|c|}{ Funcionalidad } & \multirow[b]{2}{*}{ Total } \\
\hline & & Funcional & Disfuncional leve & \\
\hline \multirow[t]{4}{*}{$\overline{\text { Edad }}$} & $18-27$ & 2 & 6 & 8 \\
\hline & $28-37$ & 4 & 6 & 10 \\
\hline & $38-47$ & 1 & 1 & 2 \\
\hline & $48-57$ & 0 & 1 & 1 \\
\hline Total & & 7 & 14 & 21 \\
\hline
\end{tabular}

Mediante la recolección de datos a los 21 pacientes se determina que el mayor número de pacientes se encuentran entre las edades de 28 a 37 años donde su mayoría representa un nivel disfuncional leve, así también hay un alto índice entre las edades de 18 a 27 años, quedando excluido el rango disfuncional grave, edades que representan adultez media y adultez joven, edades productivas con capacidades de reconocer factores de productividad funcionales o disfuncionales que modifican su dinámica familiar.

Tabla 8. Cruzada de datos de nivel académico y nivel de funcionalidad de los internos del Centro Especializado en Tratamiento de alcohol y drogas “CENTRADRO” 2021

\section{Tabla cruzada Funcionalidad*Nivel Académico}

\begin{tabular}{|c|c|c|c|c|}
\hline & \multicolumn{3}{|c|}{ Nivel Académico } & \multirow[b]{2}{*}{ Total } \\
\hline & $\begin{array}{c}\text { Educación } \\
\text { Básica }\end{array}$ & Bachiller & Nivel Superior & \\
\hline Funcionalidad Funcional & 0 & 5 & 2 & 7 \\
\hline
\end{tabular}




\begin{tabular}{|c|c|c|c|c|c|}
\hline & Disfuncional leve & 3 & 8 & 3 & 14 \\
\hline Total & & 3 & 13 & 5 & 21 \\
\hline
\end{tabular}

Mediante la recolección de datos a los 21 pacientes se determina que el mayor número de participantes presentan un nivel disfuncional leve con 8 pacientes ocupando el rango de bachilleres de la república del Ecuador, 3 con educación básica, así también 3 con educación superior, mientras que, dentro del nivel funcional, 5 pacientes son bachilleres y 2 tienen educación superior, quedando al igual que en la disfuncionalidad leve un porcentaje superior en pacientes que han cursado el bachillerato, tanto el consumo de sustancias como el nivel de funcionalidad no tienen relación especifica con algún tipo de educación, por los resultados obtenidos se observa como tanto el consumo de sustancias como el nivel de funcionalidad se encuentran repartidos en todos estos rangos.

\section{Discusión}

Estos resultados guardan comparación con lo que mencionan (Alejandra Carreño et al., 2014) Estas autoras expresan dentro de los resultados obtenidos en su investigación a 8 pacientes drogodependientes la mayoría obtuvo el nivel más alto de funcionalidad a esto expresan:

“Apgar Familiar" es Normofuncional, por lo tanto, demuestra que los sujetos se encuentran en un estado de dinámica familiar acorde a lo normal, por lo cual, no se presentan síntomas de disfunción en los integrantes que componen la familia

lo que guarda relación con los resultados obtenidos en uno de los Centros especializados en el tratamiento del alcohol y drogas ya que el mayor número de pacientes obtuvieron el nivel más alto de funcionalidad.

Pero lo que no concuerda en el estudio actual con el de las autoras anteriormente mencionadas es con los resultados obtenidos en el centro terapéutico "CENTRADRO” que presenta un nivel disfuncional leve.

Por otro lado, dentro de su investigación estas autoras emplean el uso del genograma como herramienta para determinar los roles dentro del sistema familiar, mismos temas que en la presente investigación no se toman en consideración.

\section{Conclusiones}


Habiendo evidenciado los resultados conseguidos con la aplicación del test APGAR se ha llegado a las siguientes conclusiones:

El nivel de funcionalidad varía entre los dos centros especializados en el tratamiento de alcohol y drogas, donde el CETAD "Camino a la paz" tiene un nivel funcional mientras que el CETAD “CENTRADRO” tiene un nivel disfuncional leve.

Los pacientes del CETAD "Camino a la paz" en su mayoría presentan entre un nivel funcional a disfuncional leve siendo menores a 37 años, mientras que en el CETAD “CENTRADRO” presentan nivel disfuncional leve los menores de 37 años y mayores a 28 años ocupando la mayoría de los pacientes intervenidos.

Los pacientes del CETAD “CENTRADRO” presentan un mayor número en aquellos que son bachiller presentados entre un nivel funcional y disfuncional leve, a diferencia con el CETAD "Camino a la paz" quienes tienen su mayor número de pacientes en el nivel disfuncional leve así también con pacientes que han recibido su educación hasta bachillerato.

\section{Referencias bibliográficas}

Alegre, Y., \& Suarez, M. (2006). Instrumentos de atención a la familia: El familiograma y el Apgar familiar. https://d1wqtxts1 xzle7.cloudfront.net/34898484/Familiograma_y_APGAR_familiar.pdf?1411823783=\&resp onse-content-

disposition=inline \%3B+filename\%3DFASCICULO_CADEC_Instrumentos_de_Atencion.pdf\&Expires=161 7250880\&Signature $=\mathrm{Og} 3 \mathrm{FWDHOkr}$ -

zcYTH6AUDpA0MJ3tYlBQofch87NjQawO0vV9vtkTPW3H 8YcB s2dbNMFB008STYYBO3GdvMlzhG 6hrn89bTHQiwo-jy2Ki4T8UjKg9EoA2huXfJOTtzTFLPb3QkXXazZfgWuGjKrcUyynx6X246YlcDxPvGNtt2JZVXO0WgQc--M0YjeGK9yFaJ5nsR2bxwcPpVMgGm95bePFxPqQkp1I1aBpkaHxQdjwswhrOXN418Hx4LgcZ3JQ5--Z7TixOeSZ2P4IdkchQgIt4rx6h-jpvJZI05SYdZIYV8-djkQR00P tYeYws8Svlbqvl4iQxlO oWug_\&KeyPair-Id=APKAJLOHF5GGSLRBV4ZA

Bustos, L., \& Nossa, D. (s. f.). Aportes del Magisterio de la iglesia al concepto de funcionamiento familiar (APGAR). 42-57. 
Castillo, G., Pérez, L., \& Rábago, M. (2018). ADICCIONES DESDE UN ENFOQUE DE TERAPIA SISTÉMICA FAMILIAR: APORTACIONES DEL MODELO DE STANTON Y TODD. https://www.researchgate.net/publication/327944736_ADICCIONES_DESDE_UN_ENFOQUE_DE_TERA PIA_SISTEMICA_FAMILIAR_APORTACIONES_DEL_MODELO_DE_STANTON_Y_TODD

Cócola, F. (2018). Funcionamiento familiar y abordaje terapéutico de los trastornos por el uso de sustancias: Una revisión de la literatura sistémica. http://www.redesdigital.com.mx/index.php/redes/article/view/249

Costa, M. (2014). Funcionamiento familiar percibido en la familia y el drogodependiente en proceso de recuperación [Universidad Abierta Interamericana]. http://imgbiblio.vaneduc.edu.ar/fulltext/files/TC116709.pdf

García, M., Rivera, S., Reyes, I., \& Diaz, R. (2006). Construcción de una escala de funcionamiento familiar. https://www.redalyc.org/pdf/4596/459645449006.pdf

Jaramillo, J. (2016). Terapia familiar estructurada y sentido de comunidad dentro de una comunidad terapéutica de abuso y drogodependencias [Universidad San Francisco de Quito]. http://repositorio.usfq.edu.ec/handle/23000/6116

Luna, L. (2015). La importancia de terapia familiar estratégica breve en el proceso de rehabilitación de adolescentes con alcoholismo. [Universidaddel San Francisco de Quito]. http://repositorio.usfq.edu.ec/handle/23000/5019

Marcos Sierra, J. A., \& Garrido Fernandez, M. (2009). La terapia Familiar en el tratamiento de las adicciones . Apuntes de Psicologia , 339-362.

Moreno Alvarado, E. Y. (22 de 09 de 2016). Repositorio UTMACH. Obtenido de http://repositorio.utmachala.edu.ec/bitstream/48000/8629/1/ECUACS\%20DE00029.pdf

Nolasco Pereyra, C. (s.f.). Eucac\{on Medica Continua. Obtenido de http://www.bvs.hn/RMH/pdf/1988/pdf/Vol56-4-1988-11.pdf

Ramirez Villaseñor, M. A. (2001). Terapia Familiar y Adicciones un enfoque practico con resultados practico . Revista Internacional de Psicologia , 1-10. 
Ortega, A., Fernandez, A., \& Osorio, M. (2003). Funcionamiento familiar. Su percepción por diferentes grupos generacionales $y$ signos de alarma. http://scielo.sld.cu/scielo.php?script=sci_arttext\&pid=S086421252003000400002

Rodriguez, R. (2018). Analisis sistémico de las familias de un grupo de adictos pertenecientes a la Comunidad Casa de la misericordia en Bucaramanga. Universidad Pontifica Bolivariana.

Sanchez, K., \& Serrano, M. (2019). EFECTIVIDAD DEL PROGRAMA DE ADICCIONES CON Y SIN TERAPIA FAMILIAR SOBRE LA FUNCIONALIDAD FAMILIAR Y PREVENCIÓN DE RECAÍDAS EN PACIENTES ADICTOS [UNIVERSIDAD PRIVADA ANTENOR ORREGO]. http://repositorio.upao.edu.pe/bitstream/upaorep/4658/1/REP_MED.HUMA_KIMBERLI.S\%C3\%81NCHEZ _EFECTIVIDAD.PROGRAMA.ADICCIONES.CON.SIN.TERAPIA.FAMILIAR.SOBRE.FUNCIONALID AD.FAMILIAR.PREVENCION.RECAIDAS.PACIENTES.ADICTOS.pdf

Suarez, M. (2014). APGAR FAMILIAR: UNA HERRAMIENTA PARA DETECTAR DISFUNCIÓN FAMILIAR. http://www.scielo.org.bo/scielo.php?pid=S1726-89582014000100010\&script=sci_arttext

Zapata, M. (2009). La Familia, soporte para la recuperación de la adicción a las drogas. 2, 86-94. 